\title{
Lower GCS is Related to Poor Outcome among Acute Stroke Patients with COVID-19 in A Tertiary Referral Hospital in Indonesia
}

\author{
Mieke Actress Hanna Nelly Kembuan*, Arthur Hendrik Philips Mawuntu, Yohanna, \\ Feliana, Melke Joanne Tumboimbela
}

Neurology Department, Faculty of Medicine, Universitas Sam Ratulangi/R.D. Kandou Hospital, Jl. Raya Tanawangko No. 56, Manado, Indonesia

*Corresponding author. E-mail: dr.miekekembuan@yahoo.com

Received date: Sep 3, 2021; Revised date: Nov 12, 2021; Accepted date: Nov 16, 2021

\section{Abstract}

B ACKGROUND: The Coronavirus disease 2019 (COVID-19) pandemic has forced the health workforce to take mitigative measures such as physical distancing, screening, personal protective equipment donning, and confinement on patient care. We aimed to study the outcome of acute stroke patients with suspected, probable, or confirmed COVID-19 in a tertiary referral hospital in Indonesia during the first year of COVID-19 pandemic.

METHODS: This was a retrospective study examining all medical records of adult patients suffering from acute stroke with suspected or confirmed COVID-19 who were admitted to R.D. Kandou Hospital, Manado, Indonesia, between March 2020 to March 2021. Clinical and laboratory parameters were compared between subjects with poor and good outcomes based on Glasgow Outcome Scale (GOS),

\section{Introduction}

Coronavirus disease 2019 (COVID-19) pandemic has affected the management of stroke worldwide. The number of hospital admission for acute stroke was decreased significantly as reported by many countries worldwide. Intravenous thrombolysis therapy for acute ischemic stroke could not meet the standard time window in many cases due to the restriction protocol. The same situation also affected endovascular therapy. Protective measures such as patient screening, personal protective equipment (PPE) divided into poor outcome (GOS 1-3) and good outcome (GOS 4-5).

RESULTS: Fourty-six eligible subjects were enrolled in the study. Based on the GOS, 36 subjects (78.3\%) were admitted to the hospital with poor prognosis. On admission, the median Glasgow Coma Scale (GCS) was 11, breathlessness was found in $54.3 \%$ of subjects, fever was found in only 15 subjects $(32.6 \%)$, and the lowest oxygen saturation on admission $95 \%$. We found that GCS significantly related to outcome after controlled for other factors using the logistic regression method ( $p=0.03 ; 95 \% \mathrm{CI}=1.08-4.78)$.

CONCLUSION: Lower GCS can be used to predict poor outcome in acute stroke patients with COVID-19.

KEYWORDS: COVID-19, acute stroke, Glasgow Coma Scale, outcome, Indonesia

Indones Biomed J. 2021; 13(4): 409-17 donning, and room re-designing contribute to a longer time of intervention. Hospital wards, including stroke wards, have been converted to COVID-19 isolation wards. The remaining wards have to undergo capacity reduction. This led to reduced capacity in managing acute stroke patients. Hospital staffs were re-assigned to work in COVID-19 facilities and work on a weekly shift. The senior staffs were ordered to take a temporary leave.

On the other hand, COVID-19 infection seemed to increase incidence of stroke risk. Previous studies had reported this effect. The risk of stroke in patients with COVID-19 is seven times higher, compared to other 
respiratory infections such as influenza A-B.(1) Some studies had reported that stroke caused by COVID-19 are cryptogenic or embolic stroke. Both these conditions are estimated to have undetermined significance. $(2,3)$ The pooled incidence of acute ischemic stroke in COVID-19 patients was reported to be $1.2 \%$.(4) Although the exact pathophysiology is still under research, the protocols in many hospitals have been developed to detect early stroke or provide preventive treatment in those patients having hypercoagulation disorder. The association between stroke and COVID-19 may primarily be mediated through the systemic inflammatory state induced by SARS-CoV-2 infection, which may, in turn, lead to endothelial dysfunction and induce a procoagulant state. After the infection, massive amounts of pro-inflammatory cytokines such as interleukin (IL) and tumor necroses factor are released, specifically by the activated monocyte-derived macrophages. As a response to the release of pro-inflammatory cytokines, tissue factor (TF) is released from monocyte-derived macrophages and endothelial cells. The release of tissue factor activates extrinsic coagulation pathway. This pathway had been known to create fibrin deposition and in the end, blood clotting. Moreover, in SARS-CoV infection, there are reduction in the level of ACE-2 on cells, lead to increased in serum angiotensin II and both of these effects induce a pro-inflammatory effect. The systemic inflammatory response and cytokine storm may potentially destabilize atherosclerotic plaques and cause ischemic stroke and acute myocardial infarction.(5)

Obstacles in acute stroke management for COVID-19 patients have been studied in many countries. Some solutions have been provided such as protected stroke code, robots, and telemedicine. However, these solutions cannot be perfectly be implied in our country. Moreover, to date, no Indonesian national studies examining the characteristics and outcome of patients with stroke and concomitant COVID-19 have been conducted yet. We aimed to study the outcome of acute stroke patients with suspected, probable, or confirmed COVID-19 in a tertiary referral hospital in Indonesia and identify the existed problems.

\section{Methods}

\section{Study Design and Subjects}

This study was conducted in R.D. Kandou Hospital, Manado, a tertiary referral hospital serves as a referral hospital in North Sulawesi and nearby provinces. We examined all medical records of adult patients suffering from acute stroke with suspected or confirmed COVID-19 who were admitted to the hospital between March 2020 to March 2021. An ethical approval was obtained (Ethical Clearance No. 033/ EC/KEPK-KANDOU/III/2021).

The eligible subjects had the following criteria: clinically diagnosed with acute stroke, have been determined as suspected, probable, or confirmed COVID-19 cases during admission, and aged $>18$ years old. Initially, we studied the demographics and clinical characteristics, neurological deficits, hematological and clinical chemistry results, chest imaging findings, brain imaging features, and COVID-19 status of the enrolled subjects. The severity of the disease was divided into mild, moderate, severe, and critical. These variables then were associated with the poor and good outcomes. The type of stroke was divided into the hemorrhagic stroke, ischemic stroke, and non-specific stroke. In those who underwent brain imaging and has ischemic strokes, the Trial of ORG 10172 in Acute Stroke Treatment (TOAST) classification system was used to further describe the ischemic stroke sub-type.

\section{Glasgow Outcome Scale (GOS)}

GOS was measured to identify the outcome. GOS was stratified as follows: 1: death; 2: persistent vegetative state; 3: severe disability (need total assistance on activity); 4: moderate disability (do not need assistance on activity but need assistive tool); and 5: good (minor deficit). We divided the GOS into poor outcome (GOS 1-3) and good outcome (GOS 4-5). Furthermore, we assessed the percentage of subjects who underwent brain imaging and the interval between hospital admission and the time of conducting brain imaging in R.D. Kandou Hospital.

\section{Glasgow Coma Scale (GCS)}

GCS was measured according to the revised standard Glasgow Coma Scale. GCS was divided into three main parameters eye response (E), verbal response (V), and motor response (M). (E) was scored 1 if there was no eye opening, scored 2 if eye opened to physical stimulus, scored 3 if eye opened to voice, and scored 4 if eyes opened spontaneously. (V) was scored 1 if there was no verbal response, scored 2 for incomprehensible sounds, scored 3 for inappropriate words, scored 4 for confusing words, and scored 5 if patient was well oriented. For the (M) component, if there was no motor response the score was 1 , abnormal extension of the limbs as a response to physical stimulus was scored as 2 , abnormal flexion of the limbs as a response to physical stimulus was scored as 3, withdrawal movement as a response to physical stimulus was scored as 4 , proper localizing movement to 
physical stimulus was scored as 5 , and obeying commands was scored as 6.(6)

\section{Neutrophil to Lymphocyte Ratio (NLR)}

NLR was calculated based on complete blood count from every patient on admission. The complete blood count was run using standard industrialized commercial machine. Then the NLR was calculated by dividing the absolute neutrophil count by the absolute lymphocyte count. This calculation was done based on differential from complete blood count.(7)

\section{Data Analysis}

The analysis was made using the Statistical Package for the Social Science (SPSS ${ }^{\circledR}$ ) version 23 (IBM, Armonk, NY, USA) software. Statistical significance was tested according to data characteristics. A $p<0.05$ was determined as the limit for statistical significance. For categoric variables, we performed a chi-square test for normally distributed data and Fisher exact test for the data that is not distributed normally. For numeric variables, the normality was tested using the Shapiro-Wilk test. Normally distributed data were then analyzed using the independent T-test and the data that is not distributed normally were tested using the Mann-Whitney test. In multivariate analysis, we analyzed the variables that yielded $p<0.2$ in the bivariate analysis using logistic regression technique for the binary outcome (poor and good). The adjusted $p$-value for the multivariate analysis was also $p<0.05$ for significancy.

\section{Results}

\section{Characteristic of the Subjects}

As many as 245 acute stroke patients were admitted during the study period. Most of them were male (57.1\%). The majority of the case was ischemic stroke (53.5\%). We found 46 eligible subjects or $18.7 \%$ of total stroke cases in the same period that were admitted to our hospital (Table 1). The composition between males and females was equal. The mean age was $62.2 \pm 11.04$ years old. Male has lower mean age compared to female $(61.0 \pm$ SD $9.5 v s .63 .4 \pm 12.5$ years old). The most frequent presenting complaint was the loss of consciousness $(76.1 \%)$. The median admission GCS was 11 (Q1=9; Q3=13). That finding surpassed breathlessness that was found only in $54.3 \%$ of subjects. Interestingly, fever was found in only 15 subjects (32.6\%) with an initial median body temperature of $36.85^{\circ} \mathrm{C}(\mathrm{Q} 1=36.5$; Q3=37.2) and the lowest oxygen saturation on admission in our cohort was still 95\%. The subjects' median length of stay was 4.5 days $(\mathrm{Q} 1=1.0$; $\mathrm{Q} 3=15.2)$. Hypertension was identified as the most common comorbidity and the initial systolic and diastolic blood pressures were $161.2 \pm 30.31 \mathrm{mmHg}$ and $86.8 \pm 14.33 \mathrm{mmHg}$, respectively. None of our cases had received COVID-19 vaccination.

Three subjects had not undergone chest X-ray for various reasons. Bilateral infiltrates accounted for the most common chest X-ray finding in our cohort (58.1\%). There was an increased white blood cells (WBC) count found in most subjects and the mean WBC count was $15,300 \pm 6,365$ cells $/ \mathrm{mm}^{3}$. The neutrophil to lymphocyte ratio in males was higher than in females but it was not statistically significant. The other chemical chemistry findings were also not significant.

About a half of our cohort was initially diagnosed as "patient under surveillance" according to the previous national guidelines for COVID-19 case definitions (March 2020), which was defined as "patients with the presence of fever $\left(\geq 38^{\circ} \mathrm{C}\right)$ or history of fever, acute upper respiratory infection, and mild-to-severe pneumonia plus prior history of traveling to any area with local transmission anytime within the 14 days before symptom onset, or history of contact with confirmed cases of COVID-19". We classified the final case definition according to the recent national guidelines and found $37.0 \%$ confirmed COVID-19 cases and 28.3\% negative COVID-19 cases. Uncertainty, as represented by the "probable" and "suspected" COVID-19 cases were still found in $19.6 \%$ of cases. Those with poor prognosis (defined as GOS 1-3) accounted for $78.3 \%$ of our cohort while only $21.7 \%$ of subjects presented with a good prognosis (GOS 4-5). After May 2020, the terminologies were changed and afterward, the case definition "probable" mostly changed the definition of "patient under surveillance" (17.4\%).

We further analyze some known predictors of poor outcomes using bivariate analysis. The age, gender, white blood cells, neutrophil were not associated with poor outcome (all $p>0.05$ ). The presence of lateralization signs, hypertension, diabetes, and chronic kidney disease were also the same. However, we found out that there was a significant statistical difference in the lower GCS on admission $(p=0.01)$. The NLR values was not significantly associated with outcome (Table 2).

When analyzed in the COVID-19 confirmed cases only (17 subjects), the percentage of deceased subjects increased to $82.4 \%$. Among the confirmed COVID- 19 cases, $52.9 \%$ of subjects were classified as severe to critical cases.

In the multivariate analysis, we only included the GCS on admission, NLR, fever, cough, and natrium serum 
Table 1. Characteristics of the subjects.

\begin{tabular}{|c|c|}
\hline Variables & $\begin{array}{l}\text { Value } \\
(n=46)\end{array}$ \\
\hline Age (years), Mean \pm SD & $62.2 \pm 11.04$ \\
\hline \multicolumn{2}{|l|}{ Presenting complains } \\
\hline Fever, n $(\%)$ & $15(32.6)$ \\
\hline Cough, n $(\%)$ & $14(30.4)$ \\
\hline Breathlessness, n (\%) & $25(54.3)$ \\
\hline Headache, n $(\%)$ & $9(19.6)$ \\
\hline Seizure, $\mathrm{n}(\%)$ & $4(8.7)$ \\
\hline Loss of consciousness, $\mathrm{n}(\%)$ & $35(76.1)$ \\
\hline \multicolumn{2}{|l|}{ Stroke Type $(\mathrm{n}=46)$} \\
\hline Ischemic stroke, n (\%) & $18(39.1)$ \\
\hline Hemorrhagic stroke, n (\%) & $27(58.7)$ \\
\hline Non-specific, n (\%) & $1(2.2)$ \\
\hline \multicolumn{2}{|l|}{ Ischemic Stroke Subtype $(\mathrm{n}=18)$} \\
\hline Large-artery atherosclerosis, n (\%) & $2(11.1)$ \\
\hline Small vessel occlusion, $\mathrm{n}(\%)$ & $2(11.1)$ \\
\hline Cardio-embolism, n (\%) & $2(11.1)$ \\
\hline Other determined cause, $\mathrm{n}(\%)$ & $0(0.0)$ \\
\hline Undetermined cause, $\mathrm{n}(\%)$ & $12(66.7)$ \\
\hline \multicolumn{2}{|l|}{ Comorbidities of the subjects } \\
\hline Hypertension, n (\%) & $38(82.6)$ \\
\hline Diabetes mellitus, n (\%) & $11(23.9)$ \\
\hline Chronic kidney disease, n (\%) & $6(13.0)$ \\
\hline Length of stay (days), Median (Q1, Q3) & $4.5(1,15.3)$ \\
\hline \multicolumn{2}{|l|}{ Clinical findings on arrival } \\
\hline Systolic BP (mmHg), Mean \pm SD & $161.2 \pm 30.31$ \\
\hline Diastolic BP (mmHg), Mean \pm SD & $86.8 \pm 14.33$ \\
\hline Body temperature $\left({ }^{\circ} \mathrm{C}\right)$, Median $(\mathrm{Q} 1, \mathrm{Q} 3)$ & $36.85(36.5,37.2)$ \\
\hline Oxygen saturation (\%), Median (Q1, Q3) & $98(95,99)$ \\
\hline Total GCS, Median (Q1, Q3) & $11(9,13.3)$ \\
\hline \multicolumn{2}{|l|}{ Chest X-ray $(n=43)$} \\
\hline Normal, n (\%) & $7(16.3)$ \\
\hline Unilateral infiltrate, $\mathrm{n}(\%)$ & $9(20.9)$ \\
\hline Bilateral infiltrate, $\mathrm{n}(\%)$ & $25(58.1)$ \\
\hline \multicolumn{2}{|l|}{ Clinical chemistry results } \\
\hline Hemoglobin (g/dL), Mean \pm SD & $13.4 \pm 2.63$ \\
\hline Hematocrit (\%), Median (Q1, Q3) & $40(36.5,44.5)$ \\
\hline Platelet (cells/mm³), Median (Q1, Q3) & $229000(189250,292000)$ \\
\hline $\mathrm{WBC}\left(\right.$ cells $\left./ \mathrm{mm}^{3}\right), \mathrm{Mean} \pm \mathrm{SD}$ & $15300.0 \pm 6365.04$ \\
\hline Neutrophil (\%), Median (Q1, Q3) & $84(75.75,88.25)$ \\
\hline Lymphocyte (\%), Median (Q1, Q3) & $10.5(7,15)$ \\
\hline NLR, Median (Q1, Q3) & $8.1(4.5,11.7)$ \\
\hline RBS (mg/dL), Median (Q1, Q3) & $133(105.3,161)$ \\
\hline ALT(U/L), Median (Q1, Q3) & $36(25,56)$ \\
\hline AST (U/L), Median (Q1, Q3) & $21(14,38.5)$ \\
\hline Ureum (mg/dL), Median (Q1, Q3) & $39(24.5,61.5)$ \\
\hline Creatinine (mg/dL), Median (Q1, Q3) & $1.1(0.8,1.4)$ \\
\hline Sodium serum (mEq/L), Median (Q1, Q3) & $139.5(136,141.3)$ \\
\hline Potassium serum (mEq/L), Median (Q1, Q3) & $3.9(3.4,4.4)$ \\
\hline Chloride serum (mEq/L), Median (Q1, Q3) & $99.6(95.5,102.6)$ \\
\hline
\end{tabular}

Definition from the previous national guidelines (March 2020). BP: blood pressure; GCS: Glasgow coma scale; WBC: white blood count; NLR: neutrophil to lymphocyte ratio; RBS: random blood sugar; ALT: alanine transferase; AST: aspartate transferase. 
Table 2. Association between characteristics of the subjects and outcome.

\begin{tabular}{|c|c|c|c|}
\hline \multirow{2}{*}{ Variables } & \multicolumn{2}{|c|}{ Group } & \multirow{2}{*}{$p$-value } \\
\hline & Poor $(n=36)$ & $\operatorname{Good}(n=10)$ & \\
\hline Age (years), Mean \pm SD & $62.0 \pm 11.6$ & $63.0 \pm 9.3$ & 0.8 \\
\hline \multicolumn{4}{|l|}{ Gender } \\
\hline Male, n (\%) & $17(47.2)$ & $6(60.0)$ & \multirow{2}{*}{0.72} \\
\hline Female, n (\%) & $19(52.8)$ & $4(40.0)$ & \\
\hline \multicolumn{4}{|l|}{ Presenting complains } \\
\hline Fever, n $(\%)$ & $14(38.9)$ & $1(10.0)$ & 0.09 \\
\hline Cough, n (\%) & $9(25.0)$ & $5(50.0)$ & 0.13 \\
\hline Breathlessness, n (\%) & $19(52.8)$ & $6(60.0)$ & 0.48 \\
\hline Headache, n $(\%)$ & $8(22.2)$ & $1(10.0)$ & 0.36 \\
\hline Seizure, $\mathrm{n}(\%)$ & $3(8.3)$ & $1(10.0)$ & 0.64 \\
\hline \multicolumn{4}{|l|}{ Stroke type } \\
\hline Ischemic stroke, $\mathrm{n}(\%)$ & $11(30.6)$ & $7(70.0)$ & \multirow{3}{*}{0.05} \\
\hline Hemorrhagic stroke, $\mathrm{n}(\%)$ & $24(66.7)$ & $3(30.0)$ & \\
\hline Non-specific, n (\%) & $1(2.8)$ & $0(0.0)$ & \\
\hline \multicolumn{4}{|l|}{ Grade of disease $(n=17)$} \\
\hline Mild, n (\%) & $5(35.7)$ & $1(33.3)$ & \multirow{3}{*}{0.77} \\
\hline Moderate, n (\%) & $1(7.1)$ & $1(33.3)$ & \\
\hline Severe-critical, n (\%) & $8(57.1)$ & $1(33.3)$ & \\
\hline \multicolumn{4}{|l|}{ Comorbidities of the subjects } \\
\hline Hypertension, n (\%) & $29(80.6)$ & $9(90.0)$ & 0.44 \\
\hline Diabetes mellitus, $\mathrm{n}(\%)$ & $8(22.2)$ & $3(30.0)$ & 0.45 \\
\hline Chronic kidney disease, n (\%) & $4(11.1)$ & $2(20.0)$ & 0.39 \\
\hline Length of stay (days), Median (Q1, Q3) & $3.5(1,13.5)$ & $10.5 \pm 8.0$ & 0.39 \\
\hline \multicolumn{4}{|l|}{ Clinical findings on arrival } \\
\hline Systolic BP (mmHg), Mean \pm SD & $162.4 \pm 32.8$ & $156.8 \pm 19.5$ & 0.61 \\
\hline Diastolic BP (mmHg), Median (Q1, Q3) & $90(73.3,100)$ & $87.8 \pm 16.2$ & 0.78 \\
\hline Body temperature $\left({ }^{\circ} \mathrm{C}\right)$, Median $(\mathrm{Q} 1, \mathrm{Q} 3)$ & $36.9(36.4,37.7)$ & $36.7 \pm 0.4$ & 0.32 \\
\hline Oxygen saturation, Median (Q1, Q3) & $98(94.3,98)$ & $98(97,99)$ & 0.11 \\
\hline GCS, Mean \pm SD & $9.8 \pm 3.2$ & $14.5(13,15)$ & 0.01 \\
\hline Pupil anisochority, n (\%) & $5(13.9)$ & $0(0.0)$ & 0.28 \\
\hline Cranial nerves palsy, n (\%) & $27(75.0)$ & $9(90.0)$ & 0.29 \\
\hline Hemiparesis, $\mathrm{n}(\%)$ & $28(77.8)$ & $9(90.0)$ & 0.36 \\
\hline \multicolumn{4}{|l|}{ Clinical chemistry results } \\
\hline Hemoglobin (g/dl), Mean \pm SD & $13.6 \pm 2.7$ & $12.8 \pm 2.4$ & 0.35 \\
\hline Hematocrits (\%), Median (Q1, Q3) & $40.2(36.5,45.3)$ & $40.0(34.0,41.1)$ & 0.42 \\
\hline Platelet (cells $\left./ \mathrm{mm}^{3}\right)$, Median (Q1, Q3) & $239500(175000,312250)$ & $212000(193750,267250)$ & 0.82 \\
\hline NLR, Median (Q1, Q3) & $8.7(2.2,29.7)$ & $4.7(2.3,16.8)$ & 0.2 \\
\hline RBS, Median (Q1, Q3) & $128(108.5,159.5)$ & $138.5(100,217)$ & 0.89 \\
\hline ALT, Median (Q1, Q3) & $34(24.5,54)$ & $51(26,64)$ & 0.29 \\
\hline AST, Median (Q1, Q3) & $19(14,35)$ & $36.4 \pm 22.2$ & 0.24 \\
\hline Ureum, Median (Q1, Q3) & $39(26.5,64)$ & $38.5(23,49)$ & 0.55 \\
\hline Creatinine, Median (Q1, Q3) & $1(0.8,1.4)$ & $1.1(0.8,1.5)$ & 0.55 \\
\hline Sodium serum, Median (Q1, Q3) & $140(136.5,142)$ & $137.5(136,139)$ & 0.05 \\
\hline Potassium serum, Median (Q1, Q3) & $3.8(3.4,4.4)$ & $4.2 \pm 1.0$ & 0.49 \\
\hline
\end{tabular}

Definition from the previous national guidelines (March 2020). BP: blood pressure; GCS: Glasgow coma scale; NLR: neutrophil to lymphocyte ratio; RBS: random blood sugar; ALT: alanine transferase; AST: Aspartate transferase.

variables to be analyzed. The decision was based on the initial $p$-value of each variable $(p \leq 0.20)$ and anticipation of collinearity. We chose the best model that yield the Nagelkerke $\mathrm{R}^{2}=64.1 \%$. Based on the multivariate analysis, we found that only lower GCS could significantly predict poor outcome in patients with stroke and concomitant
COVID-19 ( $p=0.03 ; 95 \% \mathrm{CI}=1.08-4.78)$, as you can see in Table 2 and Table 3.

\section{Type of Stroke and Access to Brain Imaging}

Most of the subjects were clinically diagnosed with hemorrhage stroke (58.7\%). However, brain imaging (CT 
Table 3. Multivariat regression analysis.

\begin{tabular}{lcccc}
\hline \multirow{2}{*}{ Variables } & Adjusted & \multicolumn{2}{c}{ 95\%CI } & \multirow{2}{*}{$\begin{array}{c}\text { Adjusted } \\
\text { OR }\end{array}$} \\
\cline { 3 - 4 } & $\boldsymbol{p}$-value & Lower & Upper & 2.24 \\
\hline GCS on admission & 0.02 & 1.15 & 4.38 & 1.82 \\
O $_{2}$ saturation on admission & 0.2 & 0.6 & 5.48 & 2.41 \\
Fever & 0.66 & 0.05 & 126.46 & 2.47 \\
Cough & 0.45 & 0.23 & 26.36 & 0.95 \\
NLR & 0.68 & 0.72 & 1.23 & 0.95 \\
Natrium serum & 0.29 & 0.85 & 1.05 & 2.98 \\
Stroke type & 0.36 & 0.29 & 30.61 &
\end{tabular}

scan) was only performed in 14 subjects (30.4\%) (Table 4). From those who underwent $\mathrm{CT}$ scan examination, all subjects showed abnormalities related to either ischemic or hemorrhagic stroke. Large-artery atherosclerosis and cardio-embolism were found in four subjects (28.6\%). Intracerebral hemorrhage alone or in combination with subarachnoid hemorrhage was found in six patients (42.9\%) (Figure 1).

As mentioned above, brain CT scans were only performed in $30.4 \%$ of subjects. In those subjects, the median waiting time for performing a $\mathrm{CT}$ scan was two days $(\mathrm{Q} 1=0.5 ; \mathrm{Q} 3=13.0)$. However, waiting time is not associated with unfavorable outcomes $(p=0.92)$. All of the subjects suffered from ischemic stroke in our cohort did not underwent thrombolysis. Furthermore, all ischemic stroke patients during the study period did not underwent thrombolysis.

\section{Discussion}

As many as 245 acute stroke patients were admitted during the study period. Most of them were male (57.1\%). The majority of the case was ischemic stroke $(53.5 \%)$. This

Table 4. Characteristics of brain imaging performed on the subjects.

\begin{tabular}{lc}
\multicolumn{1}{c}{ Variables } & Value \\
\hline Brain CT scan $(\mathrm{n}=46)$ & $14(30.4)$ \\
Done, $\mathrm{n}(\%)$ & $32(69.6)$ \\
Not done, $\mathrm{n}(\%)$ & \\
\hline Brain CT scan results ( $=14)$ & $4(28.6)$ \\
Large infarction, $\mathrm{n}(\%)$ & $4(28.6)$ \\
Small infarction, $\mathrm{n}(\%)$ & $5(35.7)$ \\
Intracerebral hemorrhage, $\mathrm{n}(\%)$ & $1(7.1)$ \\
Combined hemorrhage stroke, $\mathrm{n}(\%)$ & $2(0.5,13)$ \\
\hline Waiting time (days) (n=14), Median $(\mathrm{Q} 1, \mathrm{Q} 3)$ & \\
\hline
\end{tabular}

pattern was similar to many studies on stroke epidemiology worldwide, including INTERSTROKE study which found larger proportion of ischemic stroke (66\%) compared to hemorrhagic stroke (34\%), and also SIREN study which reported $68 \%$ cases of ischemic stroke and $32 \%$ cases of hemorrhagic stroke. $(8,9)$

Most of our subjects were presented with no fever or cough. The oxygen saturation in our cohort was also normal. This means that the physicians cannot rely only on these symptoms of COVID-19 in stroke patients, mainly because most patients with stroke are usually admitted with loss of consciousness and impaired cough reflex, so that over-reliance in respiratory symptoms to predict the severity of COVID-19 should be avoided. These results were also in line with many previous studies which reported the insignificance of these mild respiratory symptoms on admission, such as cough, in predicting the severity of COVID-19. Clinical manifestations of COVID-19 which could predict the disease severity include (but not limited to) dyspnea, hemoptysis, anorexia, diarrhea, fatigue, and abdominal pain.(10) However, to better predict the severity of COVID-19, it is necessary to take into account not only the symptoms, but also the physical and laboratory findings of patients with COVID-19.

Moreover, we did not find any difference in presenting complaints between two groups with different prognoses, meaning that the clinical manifestations of COVID-19 alone could not predict the prognosis in patients with stroke and concomitant COVID-19.

The percentage of deceased subjects was $47.8 \%$. This percentage was higher than the percentage in patients with ischemic stroke and concomitant COVID-19 where there was a reported a mortality rate of $29.2 \%$.(11) Those with poor prognosis (defined as GOS 1-3) accounted for 78.3\% of our cohort while only $21.7 \%$ of subjects presented with a good prognosis (GOS 4-5). This number was relatively similar to the previous study in 2020 that found $74.8 \%$ of 

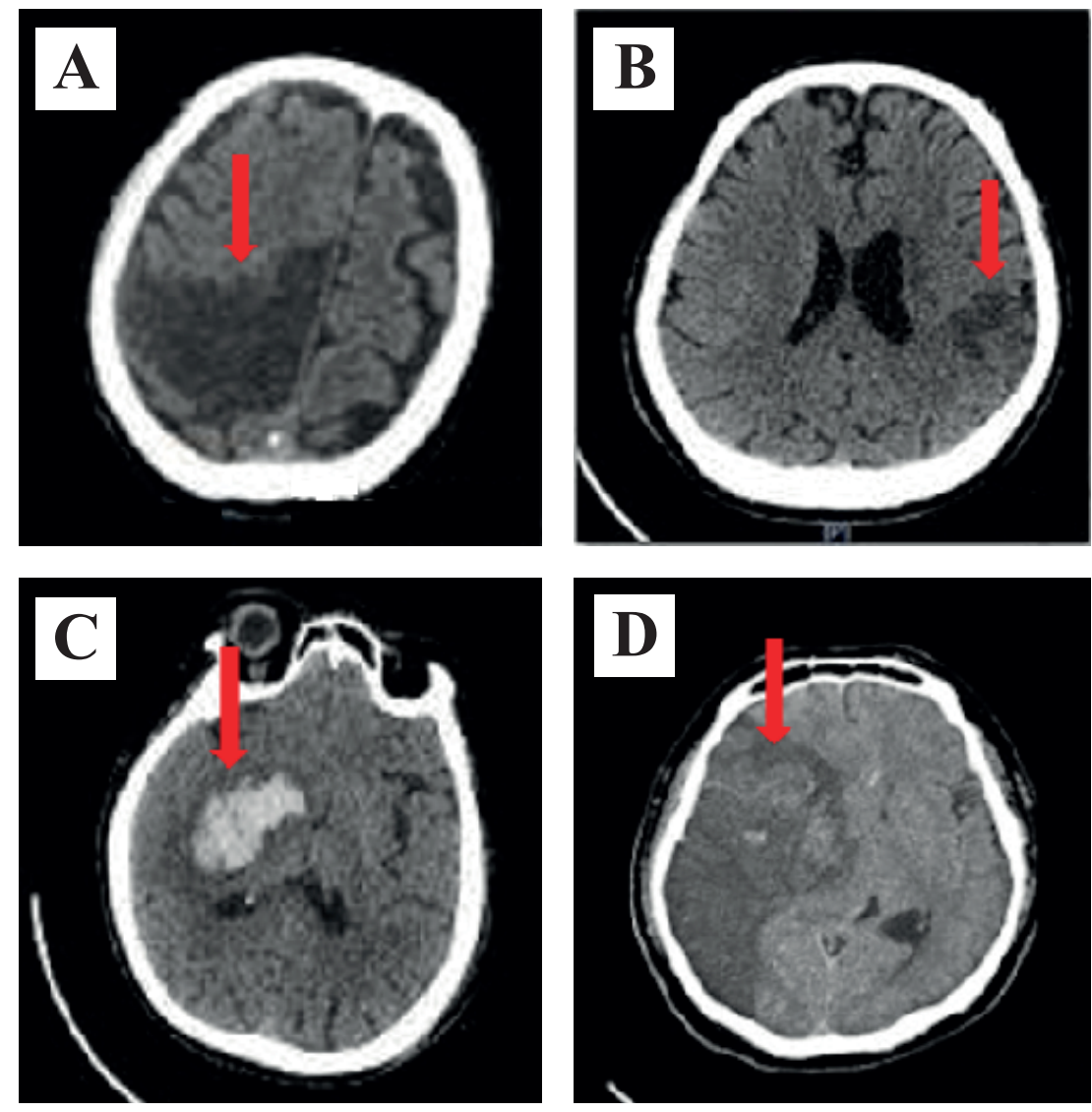

Figure 1. Examples of axial non-contrast brain CT scan performed. A: a large well demarcated hypodensity in the medial area of the right parietal region (red arrow), consistent with ischemic stroke from the occlusion of the right anterior cerebral; B: Small infarction type (diameter 2-15 $\mathrm{mm})$; $\mathrm{C}$ : a fine border hyperdensity in the right ganglia basalic (red arrow), consistent with intracerebral hemorrhage type; $\mathrm{D}$ : combined hemmorhage stroke.

patients with stroke and concomitant SARS-CoV-2 infection were critically ill.(12) The relatively higher mortality rate in our study may partly be due to the fact that this study was conducted in a tertiary hospital, where the majority of patients admitted to our hospital had multiple comorbidities which might worsen their prognosis.

Up to this time, there is no previous study that examined the role of GCS in predicting the prognosis in patients with stroke and concomitant COVID-19. However, there that encephalopathy and ischemic or hemorrhagic stroke are major neurological manifestations of COVID-19. (6) Moreover, some study observed higher mean NIHSS score in patients with stroke and concomitant SARS-CoV-2 infection compared to cases without COVID-19.(13) Further studies may be warranted to examine the diagnostic role of GCS in predicting the prognosis in patients with stroke and concomitant COVID-19.

Based on the analysis of laboratory parameters, we found that NLR was not associated with outcomes. This was confirmed when NLR was analyzed in the multivariate analysis. We assume that the influence of NLR is strongly influenced by other factors in our study. The role of NLR in COVID-19 has widely been discussed in many previous studies. In 2020, there were the first to discover the potential role of NLR in predicting the poor clinical outcome in
COVID-19 patients. Neutrophil act as major component in immune response, one of the most common leukocytes found in human body. Neutrophil could migrate from the circulation to target organ and attack viruses by releasing reactive oxygen species. This action could induce DNA damage and releasing the virus from the cells. On the other hand, systemic inflammation response significantly depresses cellular immunity, decreases $\mathrm{CD} 4^{+} \mathrm{T}$ lymphocytes, and increases $\mathrm{CD}^{+}$suppressor $\mathrm{T}$ lymphocytes. Thus, the NLR may increase in patients with COVID-19.(14) The potential role of NLR to predict the prognosis of COVID-19 has also been demonstrated by various studies in Indonesia and also outside Indonesia.(15-17) In the other study, median NLR at admission was higher among subjects with moderate to severe symptoms than the subjects with mild symptoms [6.54 (2.80-97.00) vs. 2.27 (0.79-5.07); $p<0.001]$.(15) Similar findings are also observed in another study, where the median NLR at admission is also higher among severe cases compared with non-severe cases (5.6 vs. 3.0; $p=0.007)$.(17) Moreover, NLR has excellent accuracy in predicting the severity of COVID-19, with AUC of 0.856 and optimal cut-off value of 3.17.(16) However, to the best of our knowledge, there was no previous study that examined the role of NLR in patients with stroke and concomitant COVID-19 in Indonesia. 
As for the comorbidities, in our cohort we found that most of our patients already have hypertension. Hypertension is also the risk factor of stroke, so this comorbid is usually found between COVID-19 and stroke. Hypertension as the most common comorbidities of COVID-19 patients were also found by Research Square in one of the earliest meta-analysis of COVID-19. In another analysis hypertension was the most common comorbidities followed by cardiovascular and cerebrovascular conditions and diabetes.(18)

The median length of stay in our cohort was 4.5 days. This result is similar with a systematic review in the early phase of COVID-19 pandemic, the median length of stay of COVID-19 in China was 4-53 days and the median length of stay outside China is 4 to 21 days.(19) The variative data is possible due the severity of disease. The early phase of COVID (early 2020s) are thought to be the most severe phase of COVID-19 worldwide due to unfamiliarity with the disease and there was no standard treatment.

Bilateral infiltrates on chest X-ray was our most common findings. This result is similar to early study examining COVID-19 X-ray.(20) This study found that consolidations is the most common findings and the consolidations tend to be on bilateral, peripheral, and lower zone distribution. This result is also similar with other viral pneumonia.

From clinical examination most patients from our cohort were diagnosed with hemorrhagic stroke, and only small amount of patients underwent head CT scan for confirmation due to protocols of COVID-19 treatment. A Study found that out of 2050 patients with confirmed COVID-19 infection only 21 patients (1.02\%) presented with acute ischemic stroke and 4 patients $(0.2 \%)$ presented with intracranial hemorrhage.(21) Due to limitations of diagnostic effort, every patient in our cohort were not treated with thrombolysis. But in some study, most patients underwent endovascular treatment.(21)

During the COVID-19 pandemic, some mitigative measures in the ER have hampered access to CT scan. In our cohort, almost two-thirds of the subjects could not access CT scan. This situation was caused mainly by two factors. The most common cause is the initial COVID-19 screening or chest X-ray resulted in suspected to probable COVID-19 or the patients have already been diagnosed as suspected to confirmed COVID-19 cases upon arrival in the ER. Less commonly, the patients' condition was severe and needed immediate therapeutic intervention. In those situations, the CT scan room cannot be used for them and the patients were assigned to the isolation room. The diagnosis of stroke will rely upon the clinical ground. As a consequence, no thrombolysis therapy was administered in our cohort. Patients with ischemic stroke but have initial presentation more likely to be hemorrhagic stroke such as decreased consciousness, headache, and high blood pressure will have more delay in receiving acetylsalicylic acid (ASA). On the other hand, hemorrhagic stroke patients who needed urgent neurosurgical treatment also experienced a delay in surgical intervention.

\section{Conclusion}

In conclusion, almost $20 \%$ of stroke cases in our hospital has been defined as suspected to confirmed COVID-19 cases during the first year of the pandemic. Stroke in suspected of confirmed COVID-19 patients tend to have poor prognostic and outcomes. A lower GCS may predict poor outcomes in acute stroke patients with COVID-19.

\section{Acknowledgments}

The authors thank all the participating patients and neurology residents in R.D. Kandou hospital who served during the COVID-19 pandemic.

\section{Authors Contribution}

MK, AM, and MT were involved in concepting and planning the research. AM, YO, and FE performed the data acquisition/collection. MK, AM, YO, and FE calculated the experimental data and performed the analysis. MK and AM drafted the manuscript. $\mathrm{YO}$ and $\mathrm{FE}$ designed the figures. MK, $\mathrm{AM}$, and MT aided in interpreting the results. All authors took parts in giving critical revision of the manuscript

\section{References}

1. Merkler A, Parikh N, Mir S, Gupta A, Kamel H, Lin E, et al. Risk of ischemic stroke in patients with covid-19 vs patients with influenza. JAMA Neurol. 2020; 77: 1-7. doi: 10.1001/jamaneurol.2020.2730.

2. David Spence J, De Freitas GR, Pettigrew LC, Ay H, Liebeskind DS, Kase CS, et al. Mechanisms of stroke in COVID-19. Cerebrovase Dis. 2020; 49: 451-8.

3. Ntaios G, Pearce LA, Veltkamp R, Sharma M, Kasner SE, Korompoki $\mathrm{E}$, et al. Potential embolic sources and outcomes in embolic stroke of undetermined source in the NAVIGATE-ESUS Trial. Stroke. 2020; 51: 1797-804. 
4. Tan YK, Goh C, Leow AST, Tambyah PA, Ang A, Yap ES, et al. COVID-19 and ischemic stroke: a systematic review and metasummary of the literature. J Thromb Thrombolysis. 2020; 50: 58795.

5. Wang Z, Yang Y, Liang X, Gao B, Liu M, Li W, et al. COVID-19 Associated ischemic stroke and hemorrhagic stroke: incidence, potential pathological mechanism, and management. Front Neurol. 2020; 11: 571996. doi: 10.3389/fneur.2020.571996.

6. Salahuddin H, Afreen E, Sheikh IS, Lateef S, Dawod G, Daboul J, et al. Neurological predictors of clinical outcomes in hospitalized patients with COVID-19. Front Neurol. 2020; 11: 585944. doi: 10.3389/fneur.2020.585944.

7. Yildiz H, Castanares-Zapatero D, Pierman G, Pothen L, De Greef J, Nana FA, et al. Validattion of neurophil-to-lymphocyte ratio cutoff value associated with high in-hospital mortality in COVID-19 patients. Int J Gen Med. 2021:14: 5111-7. doi: 10.2147/JJGM. S326666.

8. O'Donnell MJ, Denis X, Liu L, Zhang H, Chin SL, Rao-Melacini P, et al. Risk factors for ischaemic and intracerebral haemorrhagic stroke in 22 countries (the INTERSTROKE study): A case-control study. Lancet. 2010; 376: 112-23.

9. Sarfo FS, Ovbiagele B, Gebregziabher M, Wahab K, Akinyemi R, Akpalu A, et al. Stroke among young West Africans: Evidence from the SIREN (stroke investigative research and educational network) large multisite case-control study. Stroke. 2018; 49: 1116-20.

10. He X, Cheng X, Feng X, Wan H, Chen S, Xiong M. Clinical symptom differences between mild and severe COVID-19 patients in China: a meta-analysis. Front Public Heal. 2020; 8: 561264. doi: 10.3389/ fpubh.2020.561264.

11. Parsay S, Vosoughi A, Khabbaz A, Sadigh-Eteghad S. The incidence and mortality ratio of ischemic cerebrovascular accidents in COVID-19 cases: a systematic review and meta-analysis. J Stroke Cerebrovasc Dis. 2021; 30: 105552. doi: 10.1016/j.jstrokecerebrov asdis.2020.105552.

12. Bhatia R, Pedapati R, Komakula S, Padma Srivastava MV, Vishnubhatla S, Khurana D. Stroke in coronavirus disease 2019: A systematic review. J Stroke. 2020; 22: 324-35.

13. Perry RJ, Smith CJ, Roffe C, Simister RJ, Narayanamoorthi S, Marigold R, et al. Characteristics and outcomes of COVID-19associated stroke: A UK multicentre case-control study. J Neurol Neurosurg Psychiatry. 2021; 92: 242-8.

14. Yang AP, Liu JP, Tao WQ, Li HM. The diagnostic and predictive role of NLR, d-NLR and PLR in COVID-19 patients. Int Immunopharmacol. 2020; 84: 106504. doi: 10.1016/j.intimp.2020.106504.

15. Fuad M, Oehadian A, Prihatni D, Marthoenis M. Neutrophilto-lymphocyte ratio and Covid-19 symptom-based severity at admission. Althea Med J. 2021; 8: 1-6.

16. Selanno Y, Widaningsih Y, Esa T, Arif M. Analysis of neutrophil lymphocyte ratio and absolute lymphocyte count as predictors of severity of COVID-19 patients. Indones J Clin Pathol Med Lab. 2021; 27: 184-9.

17. Ciccullo A, Borghetti A, Zileri Dal Verme L, Tosoni A, Lombardi F, Garcovich M, et al. Neutrophil-to-lymphocyte ratio and clinical outcome in COVID-19: a report from the Italian front line. Int J Antimicrob Agents. 2020; 56: 106017. doi: 10.1016/j. ijantimicag.2020.106017.

18. Paudel SS. A meta-analysis of 2019 novel coronavirus patient clinical characteristics and comorbidities. Research Square. 2020 [PrePrint]. doi: 10.21203/rs.3.rs-21831/v1.

19. Rees EM, Nightingale ES, Jafari Y, Waterlow NR, Clifford S, Pearson CAB, et al. COVID-19 length of hospital stay: a systematic review and data synthesis. BMC Med. 2020; 18: 270. doi: 10.1186/s12916020-01726-3.

20. Cozzi D, Albanesi $\mathrm{M}$, Cavigli $\mathrm{E}$, Moroni $\mathrm{C}$, Bindi $\mathrm{A}$, Luvarà $\mathrm{S}$, et al. Chest X-ray in new Coronavirus Disease 2019 (COVID-19) infection: findings and correlation with clinical outcome. Radiol Med. 2020; 125: 730-7.

21. Requena M, Olivé-Gadea M, Muchada M, García-Tornel Á, Deck M, Juega J, et al. COVID-19 and stroke: incidence and etiological description in a high-volume center. J Stroke Cerebrovasc Dis. 2020; 29: 105225. doi: 10.1016/j.jstrokecerebrovasdis.2020.105225. 